\title{
AN EASY-TO-IMPLEMENT COMPUTATIONAL METHOD FOR THE SPACECRAFT SINE TEST-PREDICTION WITH PARTICULAR EMPHASIS ON THE MODAL CONTRIBUTIONS
}

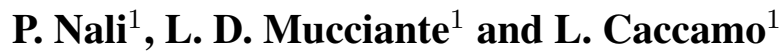 \\ ${ }^{1}$ Thales Alenia Space Italy \\ Strada antica di Collegno, 253 - 10146, Turin, Italy \\ e-mail: \{pietro.nali,luigi.mucciante,luca.caccamo\}@thalesaleniaspace.com
}

Keywords: Sine Tests, Structural Dynamics, Base-motion, Interface Forces, FRA.

\begin{abstract}
A methodology useful for calculating the frequency response functions in terms of interface forces and local accelerations of a hard-mounted structure subjected to base-shake sine tests is described in this work. The structure is specifically a SC and will be called SCO1. Reference to basic structural dynamics is done. The proposed easy-to-implement procedure permits to calculate the SC interface forces to the base and the SC local accelerations by post treating the results of the hard-mounted normal mode analysis. As a consequence of that, dedicated frequency response runs are not necessary. In accordance with the mode superposition approach, the normal mode analysis outputs are processed by accounting each modal contribution independently from the others. The relevance of each mode on the SC responses is thus explicitly quantified. This can favour a focused design refinement aiming to produce the main impact on the natural frequencies and mode shapes of interest. The critical responses can thus be modified and this can produce some advantages along the preliminary definition of the sine test notching plan. A complete set of numerical result comparisons is detailed along the paper in order to assess the accuracy of the methodology presented.
\end{abstract}




\section{Introduction}

Low-frequency base-shake sine tests, hereafter shortly called sine tests, are usually performed in the aerospace industry for identifying the modal characteristic, qualifying the structural design and verifying the structural integrity of a SpaceCraft (SC) [1]-[3]. The aim of the qualification sine tests is to make the SC reaching the qualification level of SC InterFace (I/F) loads and of some local accelerations of interest (typically related to subsystems or units).

The launch vehicle (LV) users manuals (e.g. [4],[5]) provide the Quasi-Static Loads (QSL) specification from which the qualification LV/SC I/F loads can be calculated [6]. The qualification LV/SC I/F loads are usually intended to be the I/F longitudinal force and lateral moments. The corresponding I/F in-line loads and overfluxes might also be verified.

The Sine Test Vibration Specification (STVS) is provided in the LV users manual and it is a conservative envelope of the launch transients. The STVS do not take into account the amplification factor existing around the SC most significant natural frequencies [6]. It follows that the SC I/F loads and local accelerations that could be reached during the sine test by applying the STVS input profile would significantly exceed the corresponding qualification values. Some limitations, called notching, are thus commonly introduced to the STVS in order to avoid overtesting. The notching can be considered as far as it does not jeopardise the aim of the test, that is, in this specific case, the SC sine test qualification. See work [7]-[9] for an overview of the main issues of overtesting during the sine tests.

For what concerns modeling and computations, the sine tests I/F load and acceleration responses are usually predicted in the numerical sine test-prediction through dedicated Finite Element (FE) Frequency Response Analysis (FRA), where the STVS is provided as input loading. See the work [10] for a complete overview of the Finite Element Method (FEM).

During the sine test-prediction, the so-called "primary notching strategy" is defined in order to limit the SC I/F loads that would be reached during the sine tests to the LV/SC I/F loads calculated from to the QSL specification. Also a "secondary notching" is typically defined in order to limit the acceleration or the load levels at some particular locations of the spacecraft. The limits used for computing the secondary notching correspond normally to the qualification levels of the interested subsystems or units.

It is understood that the numerical sine test-prediction plays a significant role in the SC qualification for low-frequency environment. Moreover, a large number of FE FRA might be iteratively performed in order to predict the SC structural responses to the STVS, when operating some SC structural design refinements. Each sine test-prediction imply three different FRA runs, that is one run for each axis of excitation. The SC FE model can involve a considerable number of Degree Of Freedoms (DOFs). As a consequence, each SC FRA might be rather time expensive and demanding in the computational point of view.

In this work, an easy-to-implement methodology useful for performing the sine test-prediction for the three axis of excitation is presented. The advantage of following the proposed approach is that dedicated FRA runs are not required, being the structure responses obtained by processing the output of the hard-mounted normal mode analysis through a code implemented by the authors in the Matlab numerical computing environment. The assumption of low sine sweep rate during test is made and the sine sweep rate effect [11],[12] is thus neglected.

Since the computational method presented is based on the mode superposition approach and each modal contribution is considered independently from the others, the relevance of each mode on the SC responses can be easily quantified. This can eventually provide some useful information when some structural design refinements are needed. Along this paper, the results 
obtained with the proposed methodology are assessed and compared with the FE FRA results obtained with MSC Nastran commercial software. Reference is made to the real case of a SC, hereafter called SC01.

\section{Base motion of SDOF system}

The complex equation for relative motion of a Single DOF (SDOF) system, when no force is applied to the mass, is given below [13].

$$
m \ddot{\bar{u}}+c \dot{\bar{u}}+k \bar{u}=c \dot{\bar{z}}+k \bar{z},
$$

where the overline indicates a complex quantity, $\bar{u}$ is the displacement of the mass, $\bar{z}$ is the displacement of the moving base and the dot denotes differentiation with respect to time. $m$, $c$ and $k$ are the mass, the coefficient of viscous damping and the spring constant, respectively. The hypothesis of linear oscillations is done.

If the base undergoes the simple harmonic motion $z=Z \cos (\Omega t)$, where $\Omega$ and $t$ indicate respectively the excitation frequency and the time, moving to the complex domain implies that $z=\operatorname{Re}(\bar{z})=\operatorname{Re}\left(Z e^{i \Omega t}\right)$, where $i=\sqrt{-1}$ is the imaginary unit. The following complex steady state response is assumed:

$$
\bar{u}=\bar{U} e^{i \Omega t}
$$

It is convenient to divide Eq. (1) by $m$ and rewrite it as

$$
\ddot{\bar{u}}+2 \zeta \omega_{n} \dot{\bar{u}}+\omega_{n}^{2} \bar{u}=2 \zeta \omega_{n} \dot{\bar{z}}+\omega_{n}^{2} \bar{z},
$$

where $\omega_{n}$ is the undamped circular natural frequency and $\zeta=\frac{c}{c_{c r}}$ is the viscous damping factor, with $c_{c r}=2 \sqrt{\mathrm{km}}$.

It is useful to rearrange Eq. (2) as

$$
\left(-\Omega^{2}+2 \Omega i \zeta \omega_{n}+\omega_{n}^{2}\right) \bar{U}=\left(2 \Omega i \zeta \omega_{n}+\omega_{n}^{2}\right) Z,
$$

so that $\bar{U} / Z$ takes the following form:

$$
\bar{U}=\operatorname{Tr}\left(\frac{\Omega}{\omega_{n}}\right)
$$

where

$$
\operatorname{Tr}\left(\frac{\Omega}{\omega_{n}}\right)=\frac{1+2 i \zeta \frac{\Omega}{\omega_{n}}}{1-\left(\frac{\Omega}{\omega_{n}}\right)^{2}+2 i \zeta \frac{\Omega}{\omega_{n}}} .
$$

The function $\operatorname{Tr}\left(\frac{\Omega}{\omega_{n}}\right)$ of Eq. (5) is commonly called transmissibility [13] and keeps its validity also for the subsequent derivatives of $\bar{U}$ and $Z$. That is, Eq. (5) is also the ratio between the acceleration imposed to the base and the acceleration felt by the mass: $\ddot{\bar{U}} / \ddot{Z}$. By multiplying the latter accelerations by $m$ it follows that Eq. (5) gives also the ratio between the interface force transmitted to the base dynamically $m \ddot{\bar{U}}=\bar{F}_{d y n}$ and the force $m \ddot{Z}=F_{s t}$ that would be transmitted to the base in the case of infinitely-stiff spring, that is in the case of static behaviour. By taking the absolute value of Eq. (5) leads to

$$
\frac{\left|\bar{F}_{d y n}\right|}{F_{s t}}=\frac{\sqrt{1+\left(2 \zeta \frac{\Omega}{\omega_{n}}\right)^{2}}}{\sqrt{\left(1-\left(\frac{\Omega}{\omega_{n}}\right)^{2}\right)^{2}+\left(2 \zeta \frac{\Omega}{\omega_{n}}\right)^{2}}} .
$$


Figs. 1 and 2 show respectively the curves of magnitude and phase of the transmissibility in Eq. (6), with varying $\zeta$.

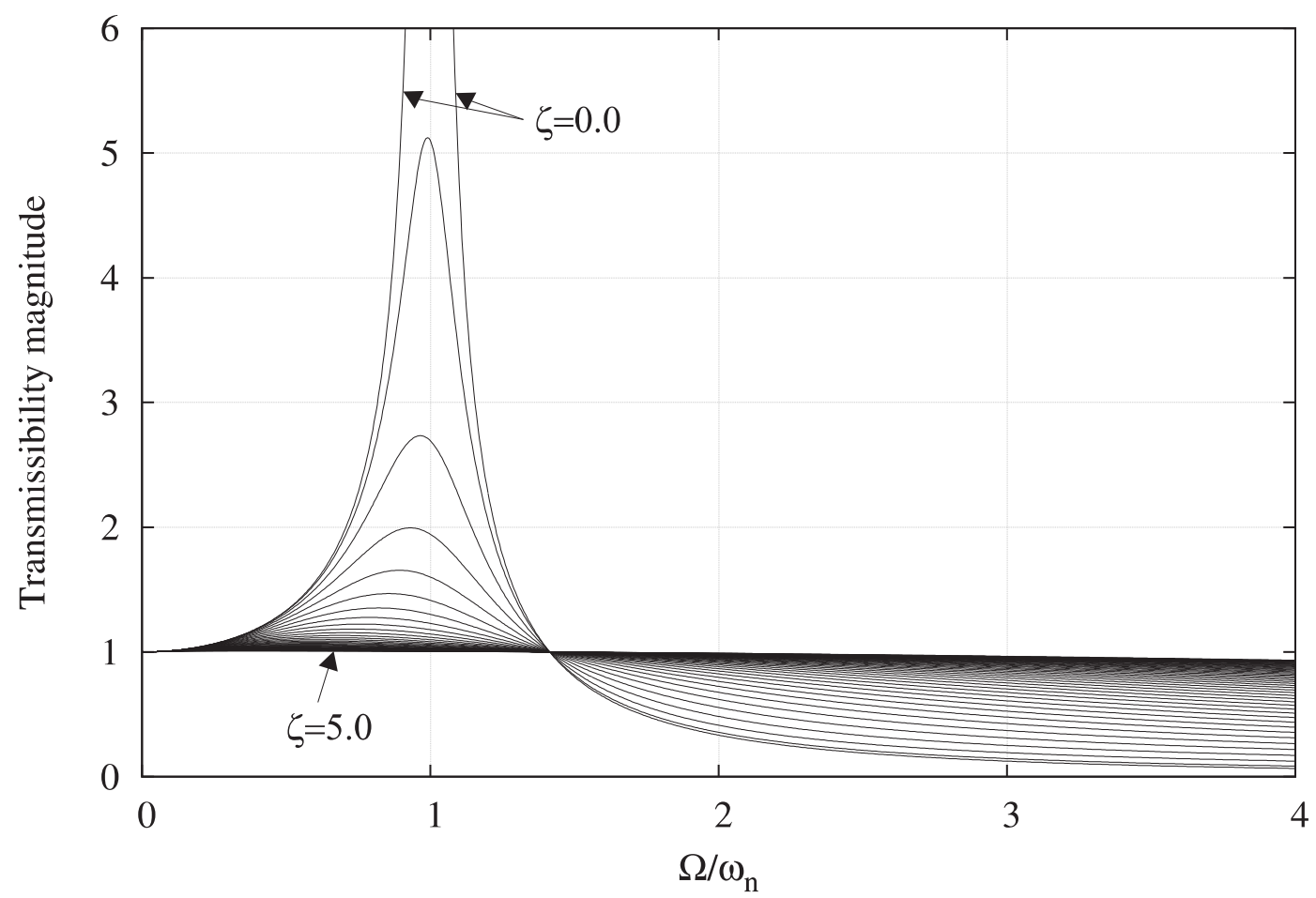

Figure 1: Transmissibility magnitude curves with $\zeta$ increasing from 0.0 to 1.5 with step of 0.1 .

\section{Base motion interface forces of mDOF systems}

The interface forces between the moving base and a multi DOF (mDOF) system may be written as

$$
\overline{\boldsymbol{F}}_{d y n}=\boldsymbol{M}_{a p p} \ddot{\overline{\boldsymbol{z}}}
$$

where bold letters denote arrays.

The apparent mass matrix $\boldsymbol{M}_{a p p}$ is usually written in the following form [3],[14]:

$$
\boldsymbol{M}_{a p p}=\boldsymbol{M}_{0}+\sum_{j=1}^{n} \boldsymbol{M}_{j}^{\mathrm{eff}} \frac{1}{-1+\left(\frac{\omega_{n j}}{\Omega}\right)^{2}+2 i \zeta_{j} \frac{\omega_{n j}}{\Omega}} .
$$

$M_{0}$ is the rigid-body mass matrix and $M_{j}^{\text {eff }}$ is the effective mass matrix associated to the $j$ th mode.

Since $\sum_{j=1}^{n} M_{j}^{\mathrm{eff}}=M_{0}$ [3], Eq. (8) becomes

$$
\boldsymbol{M}_{\text {app }}=\sum_{j=1}^{n} \boldsymbol{M}_{j}^{\mathrm{eff}}\left(1+\frac{1}{-1+\left(\frac{\omega_{n j}}{\Omega}\right)^{2}+2 i \zeta_{j} \frac{\omega_{n j}}{\Omega}}\right) .
$$

After imposing common denominator and multiplying numerator and denominator by $\left(\frac{\Omega}{\omega_{n j}}\right)^{2}$, Eq. (9) takes the form of Eq. (10), where the transmissibility ratio in Eq. (5) is recalled for the 


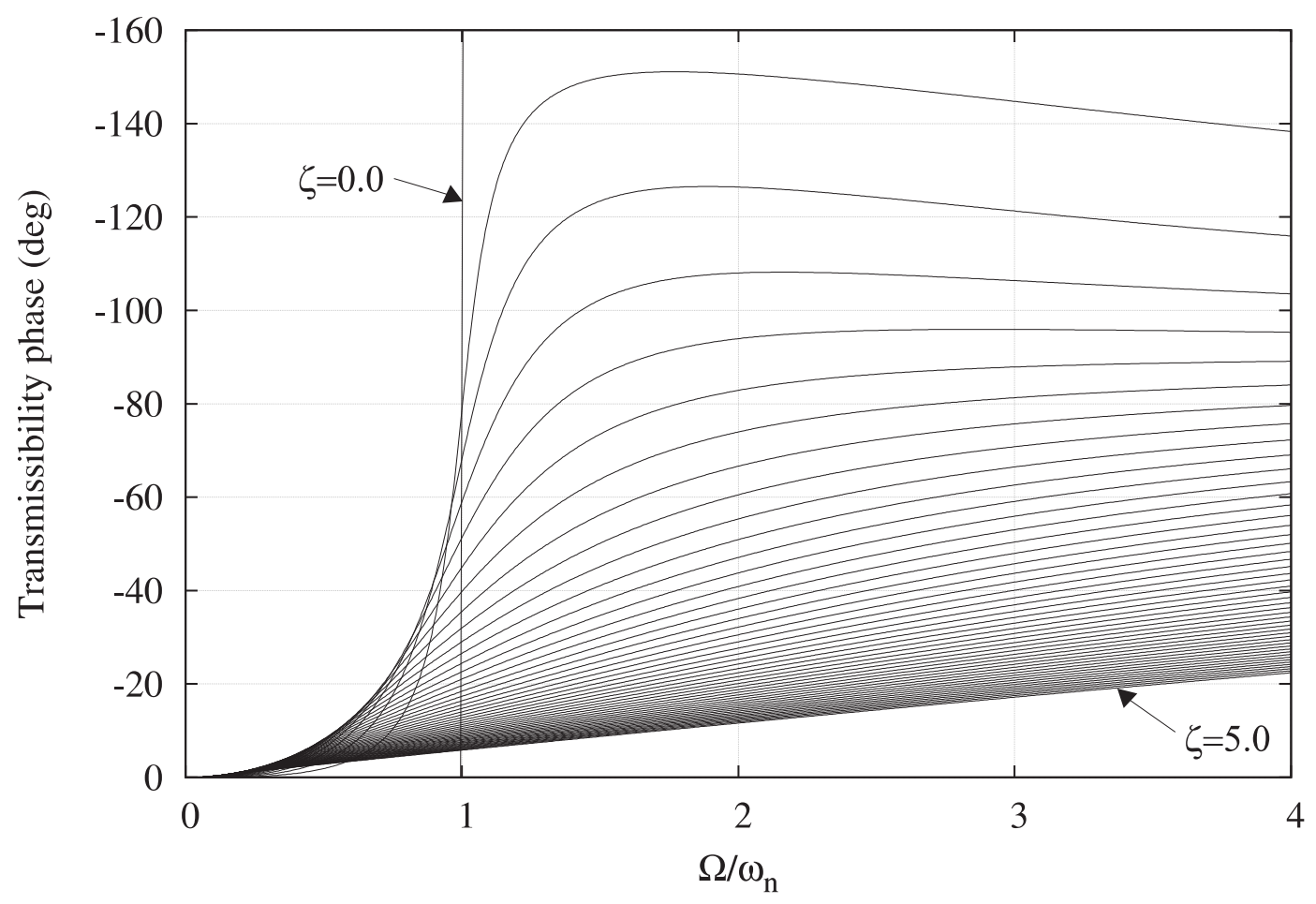

Figure 2: Transmissibility phase curves with $\zeta$ increasing from 0.0 to 1.5 with step of 0.1 .

$j$ th modal contribution.

$$
\boldsymbol{M}_{\text {app }}=\sum_{j=1}^{n} \boldsymbol{M}_{j}^{\mathrm{eff}} \operatorname{Tr}\left(\frac{\Omega}{\omega_{n j}}\right) .
$$

The effective mass residual matrix $\boldsymbol{M}_{\text {res }}^{\text {eff }}$ could be added to $\boldsymbol{M}_{\text {app }}$. In so doing, the modal contributions with $j>n$ would be considered statically when calculating the interface forces.

\section{Acceleration response of mDOF systems}

Considering the base-motion excitation vector $\ddot{\bar{z}}$, according to structural dynamics basic theory [3],[14], the displacement vector $\ddot{\bar{u}}$ is given by Eq. (11).

$$
\ddot{\overline{\boldsymbol{u}}}=\boldsymbol{\Phi} \ddot{\overline{\boldsymbol{z}}}+\sum_{j=1}^{n} \boldsymbol{\Psi}_{j} \frac{1}{m_{j j}} \frac{1}{-1+\left(\frac{\omega_{n j}}{\Omega}\right)^{2}+2 i \zeta_{j} \frac{\omega_{n j}}{\Omega}} \boldsymbol{L}_{j} \ddot{\overline{\boldsymbol{z}}},
$$

where $\boldsymbol{\Phi}$ is the rigid-body matrix, $\boldsymbol{\Psi}_{j}$ is the $j$ th fixed-base eigenvector, $m_{j j}$ is the generalized mass of the $j$ th mode and $\boldsymbol{L}_{j}$ is the $j$ th mass modal participation factor.

By assuming that the eigenvectors are mass normalized leads to $m_{j j}=1$. Moreover, by restricting the analysis only to the translational DOFs of $\ddot{\bar{u}}$, hereafter denoted with the subscript tr, Eq. (11) becomes

$$
\ddot{\overline{\boldsymbol{u}}}_{t r}=\left(\boldsymbol{\Phi}_{t r}+\sum_{j=1}^{n} \boldsymbol{\Psi}_{j} \frac{1}{-1+\left(\frac{\omega_{n j}}{\Omega}\right)^{2}+2 i \zeta_{j} \frac{\omega_{n j}}{\Omega}} \boldsymbol{L}_{j}\right) \ddot{\overline{\boldsymbol{z}}}_{t r},
$$

where the columns of $\boldsymbol{\Phi}_{t r}$ are given by the translational rigid-body modes. A rigid-body mode corresponds to the displacement vector produced by imposing the unit value to a DOF of the 
moving base and setting the remaining DOFs of the moving base to zero. It follows that the cells of $\Phi_{t r}$ are unitary or null, depending on the direction. Eq. (12) can be written in the form of Eq. (13), where the transmissibility ratio of Eq. (4) is recalled for the $j$ th modal contribution.

$$
\ddot{\overline{\boldsymbol{u}}}_{t r}=\boldsymbol{\Phi}_{t r}+\sum_{j=1}^{n} \boldsymbol{\Psi}_{j}\left(\operatorname{Tr}\left(\frac{\Omega}{\omega_{n j}}\right)-1\right) \boldsymbol{L}_{j} \ddot{\bar{z}}_{t r} .
$$

\section{Numerical results}

Within the space community, MSC Nastran is recognized as the commercial software of reference for the FEM mechanical analyses. The numerical results obtained for the sine testprediction of the SC01 are considered in this section. A Matlab code has been developed by the authors. The code reads the natural frequencies, modal effective masses, eigenvectors and mass modal participation factors from the MSC Nastran F06 file. The latter file is the output file of the hard-mounted normal mode analysis of the SC, performed with the SOL 103 of MSC Nastran. The interested responses are thus calculated by processing the information read from the F06 file, according to Eqs. 10 and 13. Several assessments are proposed in Secs. 5.1 and 5.2 in order to provide a comparison between the $\mathrm{I} / \mathrm{F}$ forces and local acceleration responses obtained with the SOL 111 of MSC Nastran (modal frequency response analysis) and the same responses calculated through the Matlab code. The responses of the longitudinal $\mathrm{I} / \mathrm{F}$ force $\mathrm{Tz}$ and the lateral I/F moments $\mathrm{Mx}$ and My are calculated for the $\mathrm{SC}$ base-excitation along the $x, y$ and $z$-axis. The $x, y$ and $z$-DOF local acceleration responses of eight nodes (mainly located in the upper zone of the SC) are calculated too. If not differently specified, the SC base-excitation is the sinusoidal input profile of 1 [g], for each axis of excitation. In Sec. 13 an example of footprint showing which modal contributions do more significantly contribute to generate $\mathrm{Tz}$ is provided and discussed. In Sec. 5.4 the notching plan of SC01 obtained by using the Matlab code is illustrated. All the responses are computed by encompassing the first 300 modal contributions. The residuals are not taken into account in the calculations. The 300th natural frequency is equal to 116.3 [Hz]. The summation of the first 300 effective masses pertaining to the translational excitation and response along the $z$-axis leads to the $95.7 \%$ of the SC mass. The frequency range from 0 to $100[\mathrm{~Hz}]$ is considered in the plots.

\subsection{I/F forces response assessment}

The frequency responses of $\mathrm{Tz}, \mathrm{Mx}$ and My I/F forces of SC01, respectively obtained with the excitation along the $z, y$ and $x$-axis, are illustrated in the Fig. 3, where the results obtained with the Matlab code are compared with those calculated with MSC Nastran. All the I/F forces curves are plotted with the frequency step of $0.05[\mathrm{~Hz}]$. A good agreement between the two set of results is confirmed in terms of magnitude. A satisfactory agreement is obtained for the response phases at low frequencies, while some differences can be detected above 60 [Hz]. The reason of such discrepancies is not clear to the authors. Additional investigations would be required in order to verify the consistency of the methodology based on Eqs. 10 and 13 with the computational process of MSC Nastran. Since the results of the sine test-prediction are not significantly sensitive to the phase differences above discussed, the agreement between the $\mathrm{I} / \mathrm{F}$ forces responses obtained with the Matlab code and those calculated with MSC Nastran is considered to be satisfactory for the purpose of this work. 

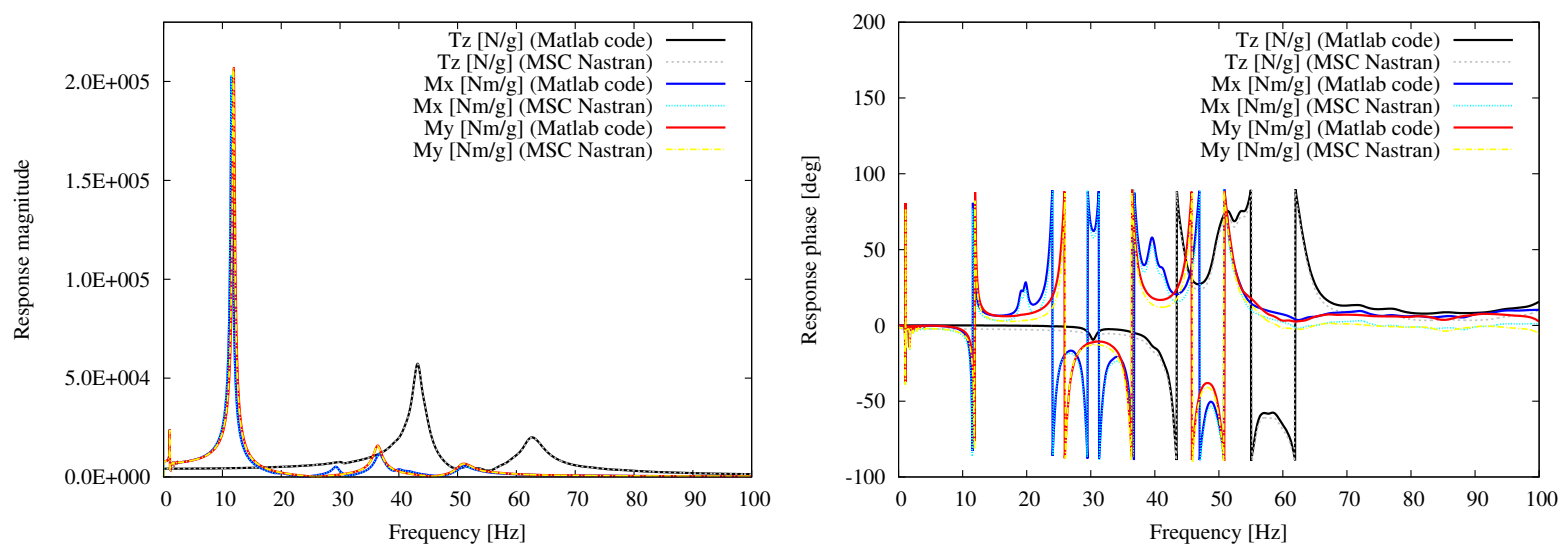

Figure 3: Frequency responses of Tz, Mx and My I/F forces of SC01: comparison of the results obtained with the Matlab code with those calculated with MSC Nastran.

\subsection{Local accelerations response assessment}

The local acceleration responses of the SC01 eight nodes under study are illustrated in Figs. 4-12. Figs. 4-6 show respectively the acceleration responses of the $x, y$ and $z$-DOFs to the excitation along the $x$-axis. Figs. 7-9 and 10-12 show the same results in case of excitation along the $y$-axis and $z$-axis, respectively. The responses calculated with the Matlab code are plotted with the frequency step of $0.1[\mathrm{~Hz}]$ while the responses obtained in MSC Nastran are plotted with the frequency step of $0.05[\mathrm{~Hz}]$. Figs. 4,8 and 12 show the in-axis responses (that is the responses of the DOFs which are parallel to the axis of excitation). Figs. 5,6,7,9,10 and 11 gives the cross-axis responses (that is the responses of the DOFs not parallel to the axis of excitation). If the magnitude plots are addressed, a good agreement is confirmed between between the results calculated with the Matlab code and those obtained in MSC Nastran. Differently, some discrepancies can be detected along the curves of the phase responses. Such differences can appear along all the considered frequency range. Concerning the reasons of such discrepancies, the comment made in Sec. 5.1 for the I/F forces responses is valid also in this case. Since the results of the sine test-prediction are not significantly sensitive to the phase differences above discussed, the agreement between the local accelerations responses obtained with the Matlab code and those calculated with MSC Nastran is considered to be satisfactory for the purpose of this work. 

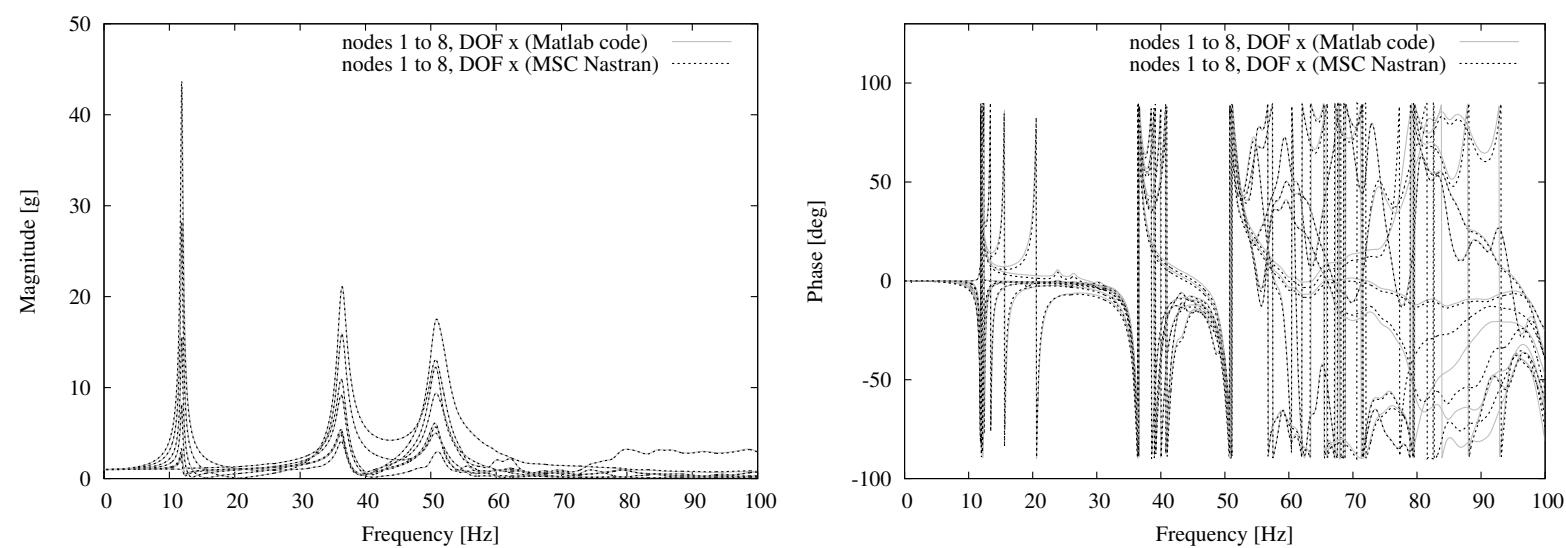

Figure 4: Frequency responses of the DOFs parallel to the $x$-axis to the sinusoidal enforced acceleration of 1 [g] along the $x$-axis.
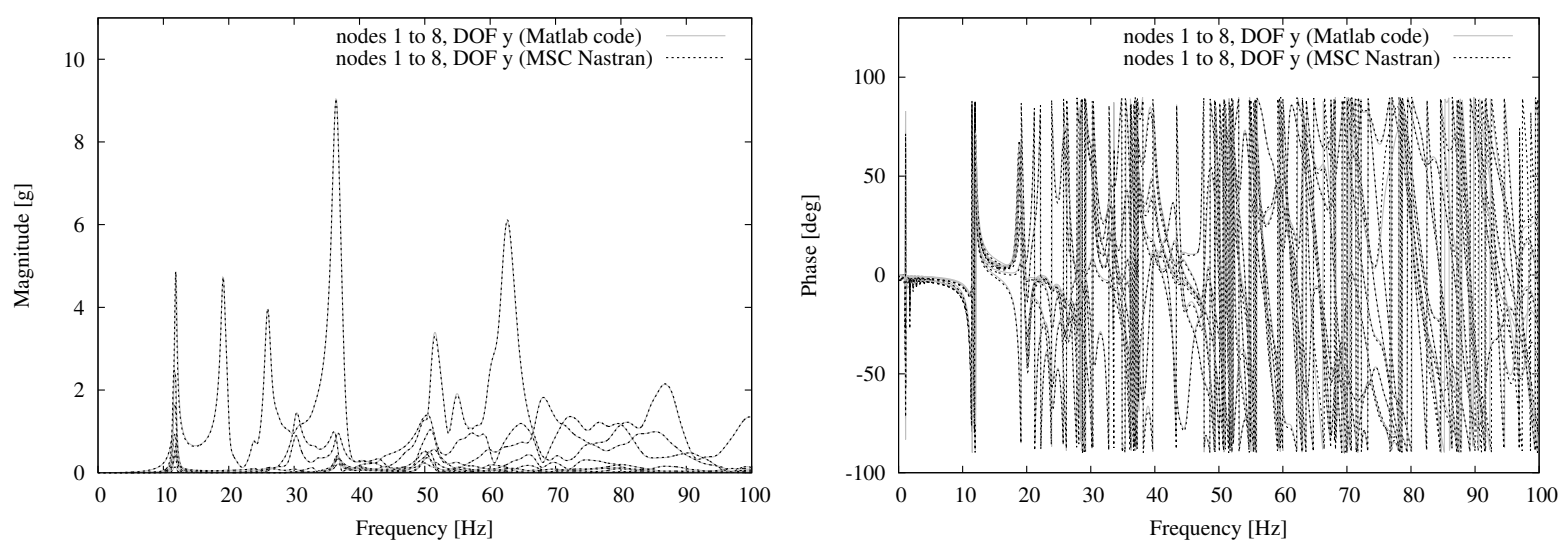

Figure 5: Frequency responses of the DOFs parallel to the $y$-axis to the sinusoidal enforced acceleration of 1 [g] along the $x$-axis.
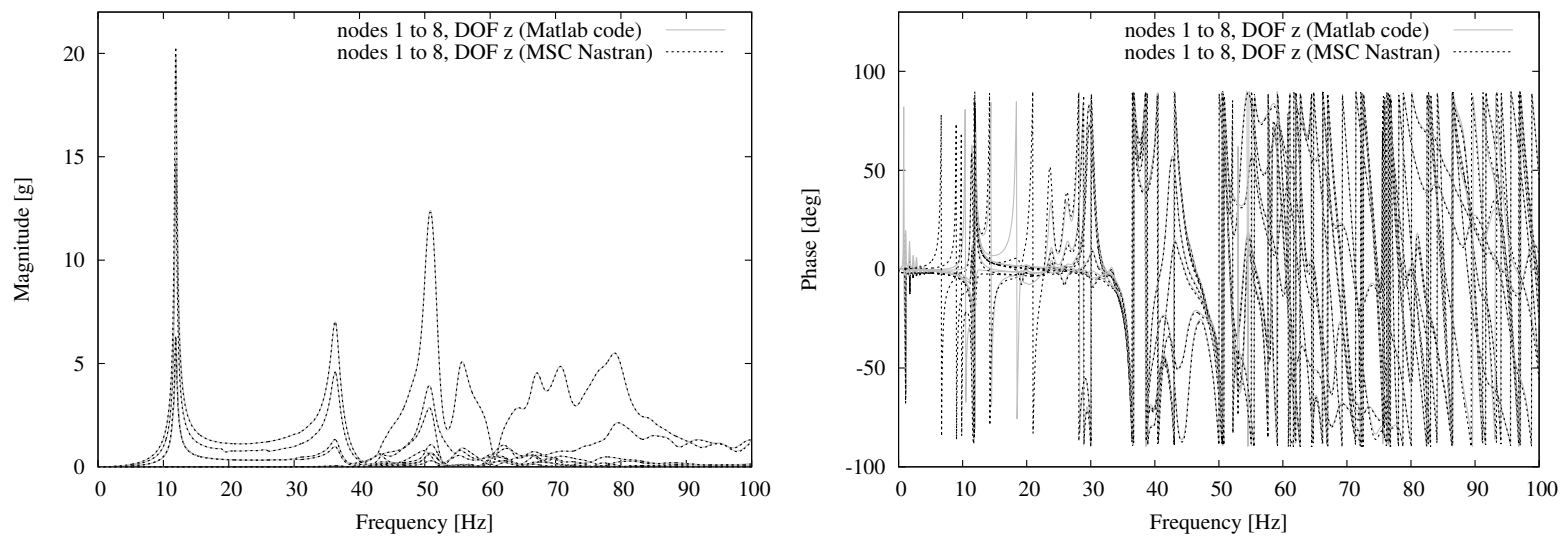

Figure 6: Frequency responses of the DOFs parallel to the $z$-axis to the sinusoidal enforced acceleration of 1 [g] along the $x$-axis. 



Figure 7: Frequency responses of the DOFs parallel to the $x$-axis to the sinusoidal enforced acceleration of $1[\mathrm{~g}$ ] along the $y$-axis.
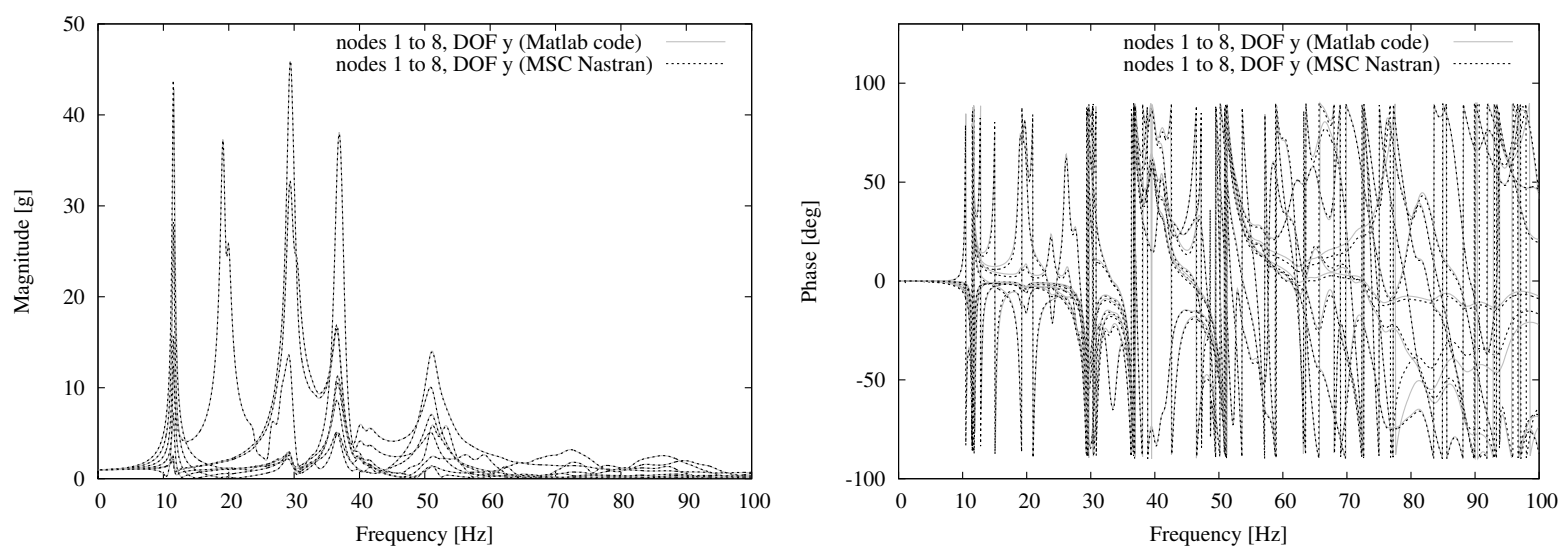

Figure 8: Frequency responses of the DOFs parallel to the $y$-axis to the sinusoidal enforced acceleration of 1 [g] along the $y$-axis.
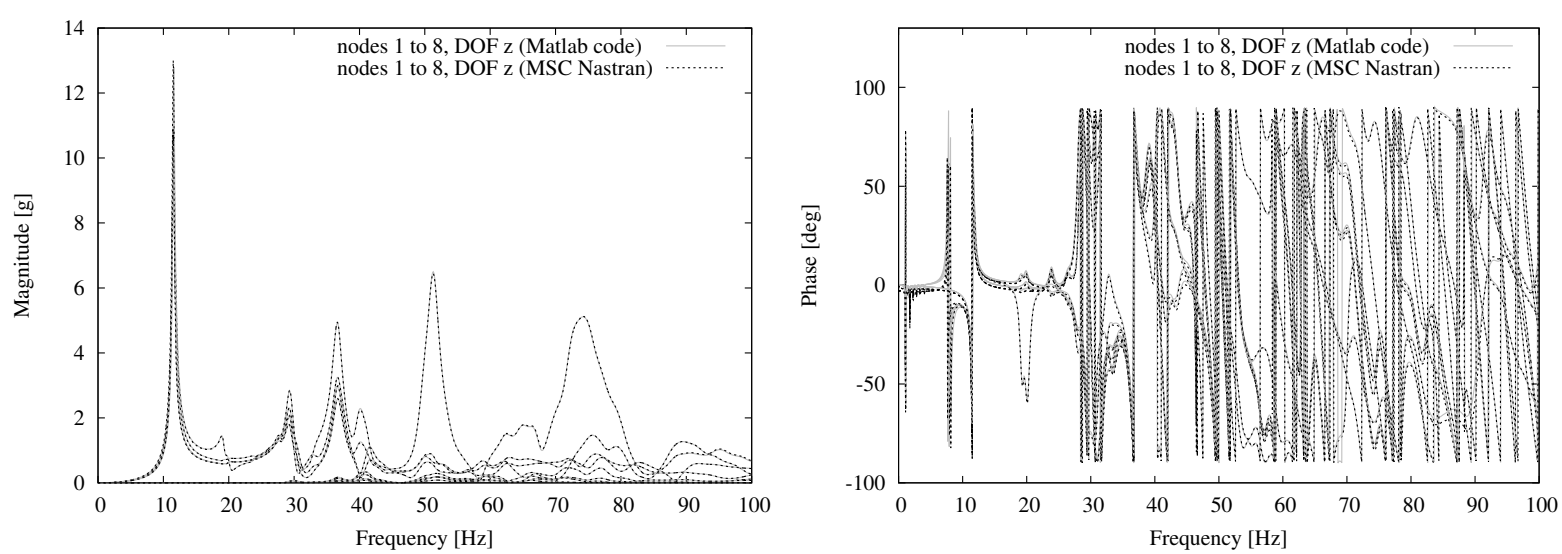

Figure 9: Frequency responses of the DOFs parallel to the $z$-axis to the sinusoidal enforced acceleration of 1 [g] along the $y$-axis. 



Figure 10: Frequency responses of the DOFs parallel to the $x$-axis to the sinusoidal enforced acceleration of $1[\mathrm{~g}$ ] along the $z$-axis.
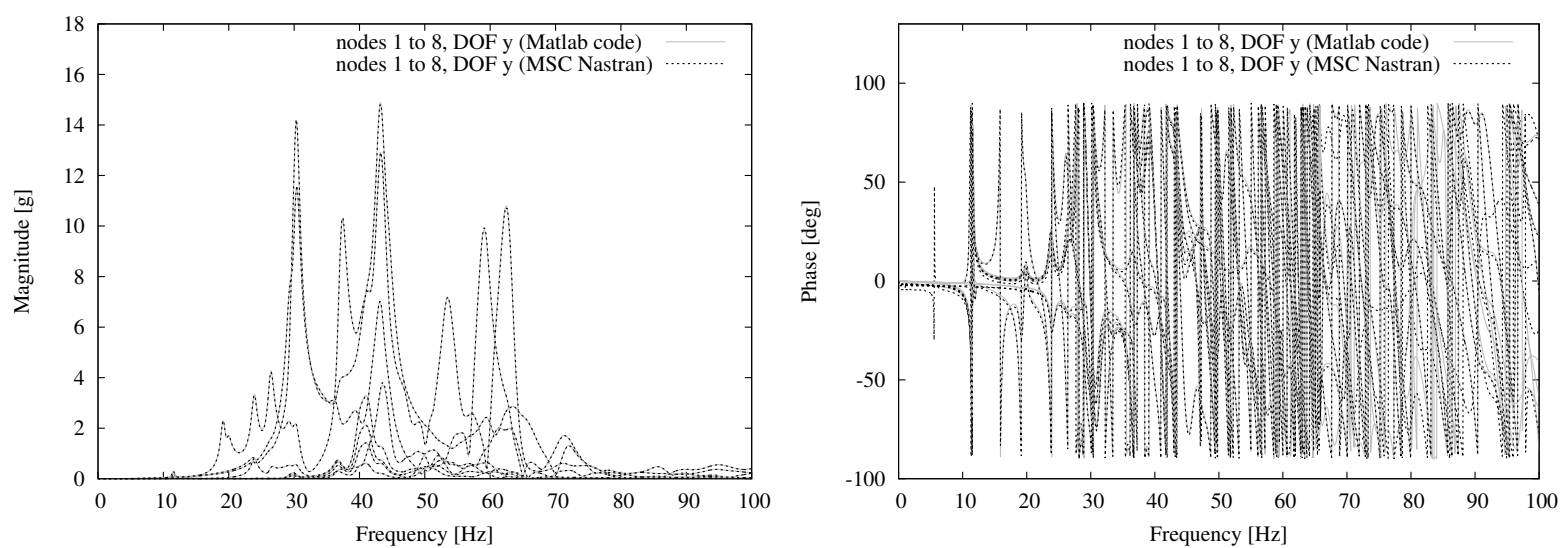

Figure 11: Frequency responses of the DOFs parallel to the $y$-axis to the sinusoidal enforced acceleration of $1[\mathrm{~g}$ ] along the $z$-axis.
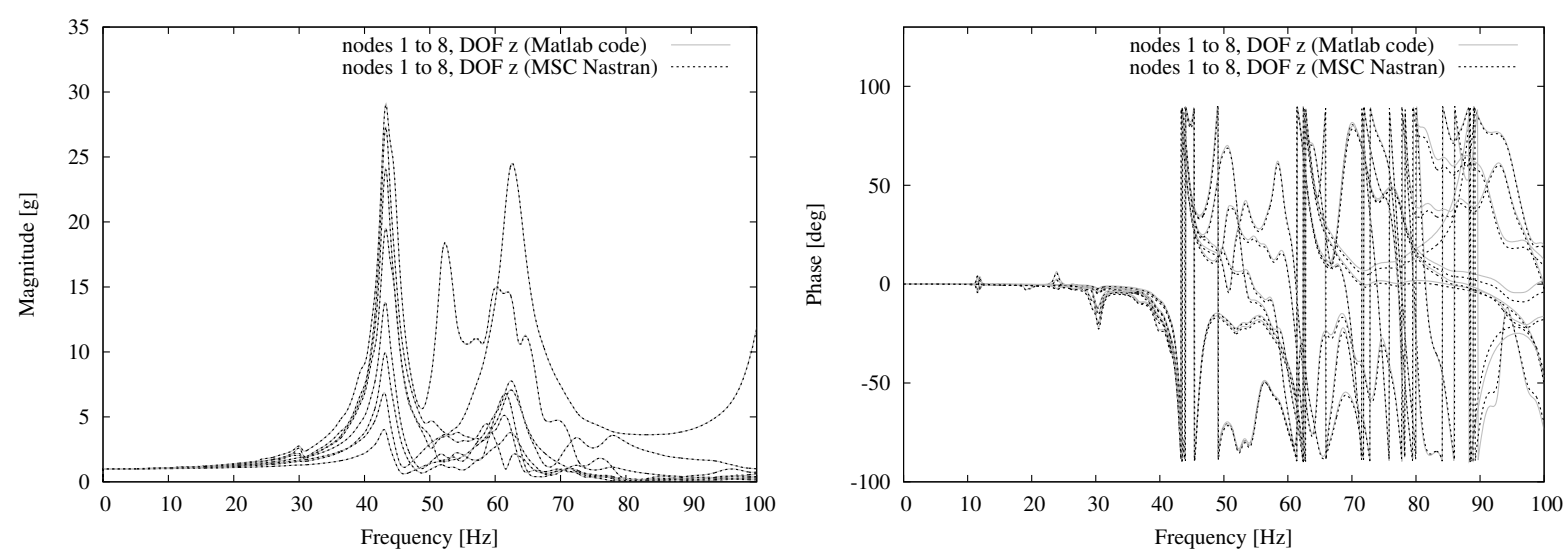

Figure 12: Frequency responses of the DOFs parallel to the $z$-axis to the sinusoidal enforced acceleration of 1 [g] along the $z$-axis. 


\subsection{Quantification of modal contributions vs frequencies}

The Fig. 13 provides some information regarding the modal contributions whose summation leads to the $\mathrm{Tz} \mathrm{I} / \mathrm{F}$ response to the sinusoidal enforced acceleration of $1[\mathrm{~g}]$ along the $z$-axis. Each point of the surface of Fig. 13 is given by the result of Eq. (7), when only the $j$ th modal contribution for a particular frequency implying a specific $\frac{\Omega}{\omega_{n j}}$ ratio is considered. The surface provides a self-explaining footprint of the modes which most significantly contribute to rise the value of the $\mathrm{Tz} \mathrm{I/F}$ force vs the corresponding frequency. The same kind of plot can be obtained for the remaining $\mathrm{I} / \mathrm{F}$ forces/moments or the local accelerations. In the latter case, Eq. (12) should be considered and the obtained footprint would reveal the modes most significantly contributing to rise the value of the interested local acceleration response vs the corresponding frequency. It is understood that such footprints can be useful in the SC design phase, when some targeted structural design refinements might are required with the aim of reducing some I/F forces and/or local acceleration responses. A critical interpretation of those surfaces might help finding design solutions which limit/shift the responses associated to particular modal contributions. In practise this could be achieved by stiffening/softening the modes of interest or simply trying to update the design in order to modify the corresponding mode shape. A few iterations may be required before freezing the design.

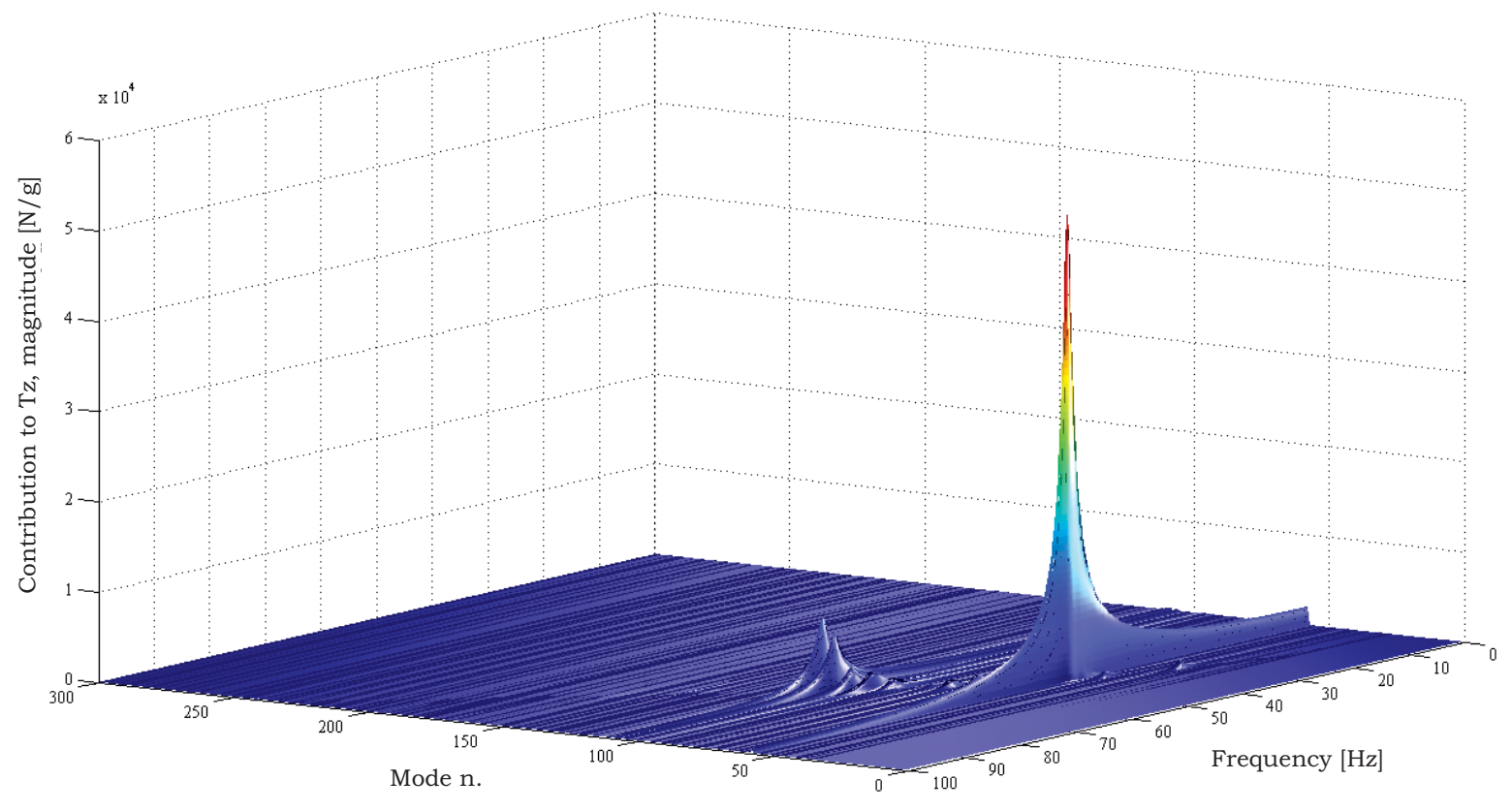

Figure 13: Surface indicating the magnitude of each modal contribution to $\mathrm{Tz}$ vs frequencies.

\subsection{The notching plan of SC01}

The sine test notching plan of SC01 is illustrated in this section for the three axis of excitation. The STVS input profile provided in the LV user manual is considered. The I/F force and local acceleration responses are calculated in accordance with the methodology proposed in this work by using the Matlab tool developed by the authors. The QSL provided in the LV user manual leads to the qualification LV/SC I/F loads. That is, the primary notching limitations are calculated for Mx, My and Tz. The secondary notching limitations are provided 
by the qualification levels of the subsystems or units associated to the interested nodes. Each node and DOF representing a subsystem or unit with a secondary notching limitation should have an appropriate sensor placed in the corresponding position of the testing structure. Fig. 14 illustrates the notched input profile for the $x$-axis. The limitation on My gives the primary notching while the limitations on local accelerations provide the secondary notching. Only one secondary notching cross-axis limitation is present (node 1 acceleration along the $z$-axis). The remaining five secondary notchings are in-line limitations (along the $x$-axis). Fig. 15 shows the notched input profile for the $y$-axis. In this case the primary notching is given by Mx while no secondary notching cross-axis limitations are present, being all the eight limitations in-line (along the $y$-axis). Fig. 16 gives the notched input profile for the $z$-axis. In this case the primary notching is given by $\mathrm{Tz}$ and three secondary notching cross-axis limitations are present (node 3,4 and 6 acceleration along the $y$-axis). The remaining seven secondary notching are in-line limitations (along the $z$-axis).

In this specific case, concerning the SC01 qualification sine tests, a manual notching enveloping both primary and secondary notches will be defined for all the three axis of excitation in order to simplify the test control.

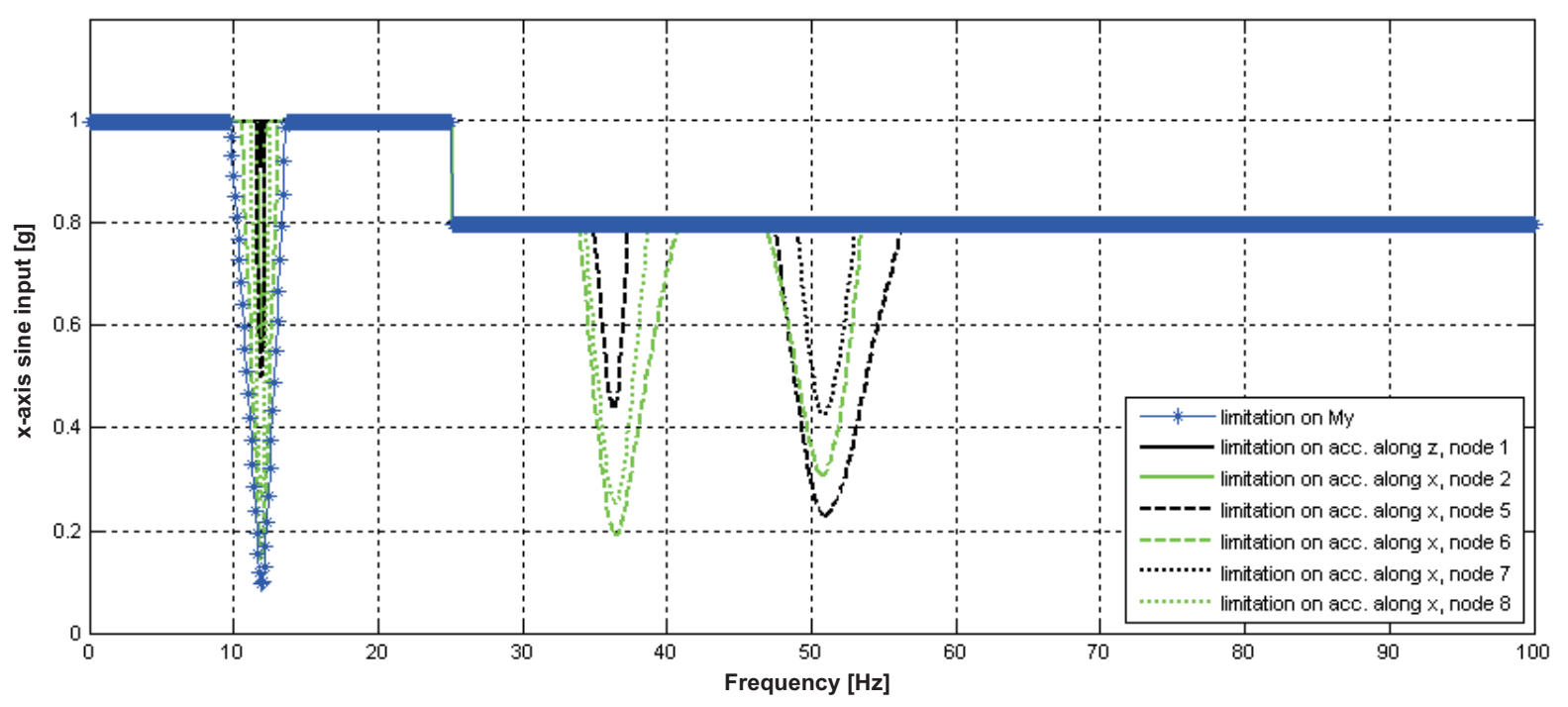

Figure 14: Primary and secondary notching profiles, $x$-axis. 




Figure 15: Primary and secondary notching profiles, $y$-axis.

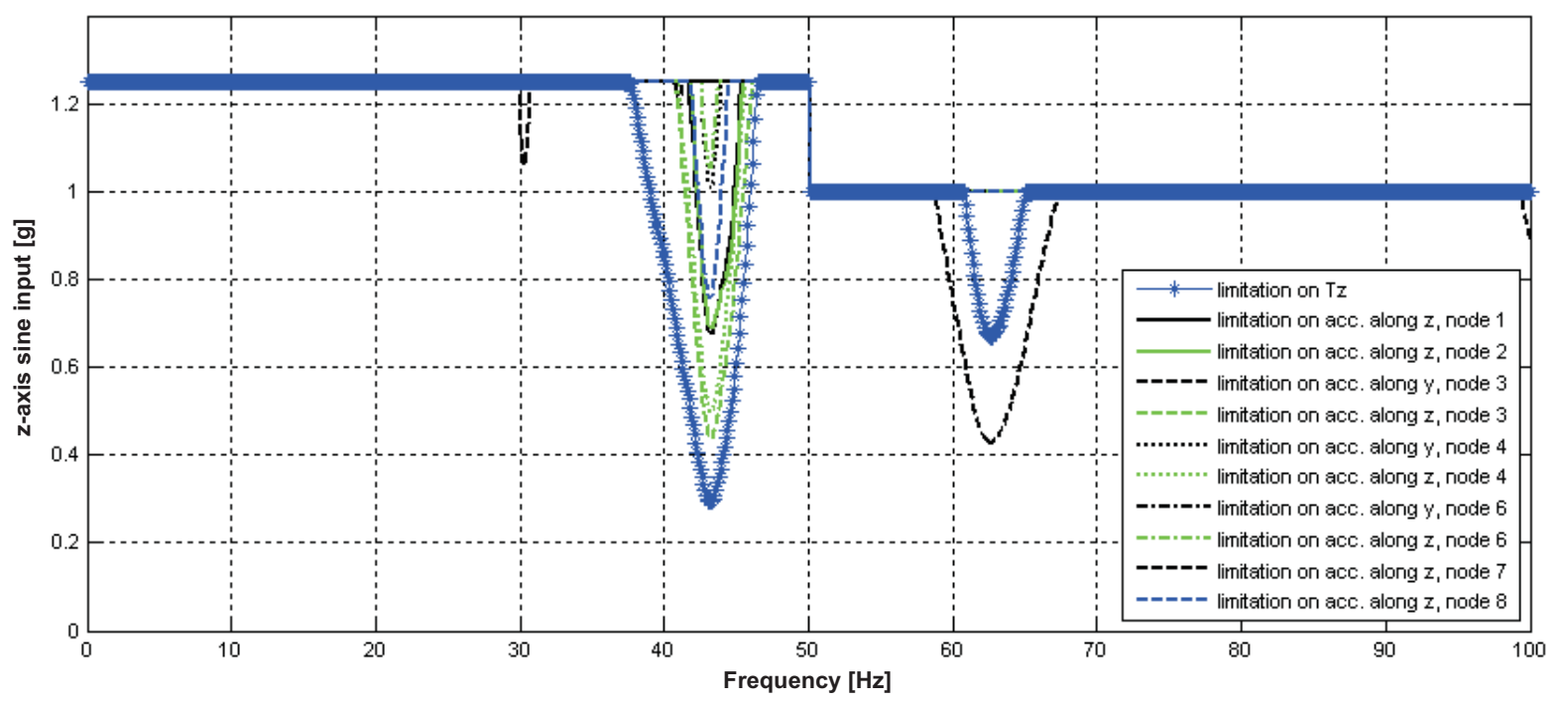

Figure 16: Primary and secondary notching profiles, $z$-axis. 


\section{Conclusions}

In this work an easy-to-implement methodology useful for calculating the frequency response functions in terms of $\mathrm{I} / \mathrm{F}$ forces and local accelerations of a hard-mounted structure subjected to base-shake sine tests was proposed. The assumption of no sine sweep rate effect was made. After a theoretical introduction recalling some basics concepts of structural dynamics, the equations useful for the implementation of the methodology discussed were obtained and commented. The implementation of the methodology described was made by the authors in Matlab. Several result assessments and comparisons with MSC Nastran FRA solutions were reported in order to confirm the accuracy of the Matlab code. The advantage of the approach presented in this work is that the frequency responses to the $x, y$ and $z$-axis sinusoidal excitations are calculated by simply processing the output of the mode analysis. The example of SC01 sine test-prediction was studied, where the SC01 frequency responses for the three directions of excitations were calculated with the Matlab code. The file provided as input was the F06 file of the normal mode MSC Nastran analysis of the hard-mounted structure of SC01. No dedicated MSC Nastran FRA runs were thus required for performing the sine test-prediction and defining the primary and secondary notching plans. An additional advantage of the methodology proposed is that the relevance of each mode on the SC responses can be explicitly quantified both for interface forces and local accelerations. This can be particularly useful in the SC design phase, when some targeted structural design refinements might be required in order to reduce some I/F forces and/or local acceleration responses.

\section{REFERENCES}

[1] C. Lalanne, Mechanical vibration \& shock analysis, Vol 1: Sinusoidal Vibrations, 2nd Edition. John Wiley \& Sons, 2009.

[2] A Girard and N. Roy, Structural dynamics in industry. ISTE Ltd and John Wiley \& Sons, ISBN: 978-1-84821-004-2, 2008.

[3] J. Wijker, Mechanical vibrations in spacecraft Design. Springer, 2004.

[4] Rockot User's Guide. Issue 05, August 2011.

[5] Ariane 5 Users Manual. Issue 05 Revision 1, July 2011.

[6] Spacecraft mechanical loads analysis handbook. ECSS-E-HB-32-26A, 2013.

[7] Y. Soucy and A Côté, Reduction of overtesting during vibration tests of space hardware. Canadian Aeronautics and Space Journal, Vol. 48, No 1, March 2002.

[8] P. Nali and A. Calvi, An investigation on the spacecraft design loads cycles and sine vibration test. TEC-MCS/2006/1362/ln/AC, ESA, Noordwijk, The Netherlands, March 2006.

[9] A. Calvi and P. Nali, Some remarks on the reduction of overtesting during base-drive sine vibration tests of spacecraft. ECCOMAS Conf. on Computational Methods in Structural Dynamics and Earthquake Engineering, Rethymno, Crete, Greece, June 2007.

[10] O.C. Zienkiewicz and R.C. Taylor, The finite element method, Vol. 1, 4th Edition. McGraw Hill, 1989. 
[11] J. A. Lollock, The effect of a swept sinusoidal excitation on the response of a singledegree-of-freedom oscillator. 43rd AIAA Structures, Structural Dynamics, and Materials Conference, Denver, Colorado, 22-25 March, 2002.

[12] N. Roy and A. Girard, Revisiting the effect of sine sweep rate on modal identification. European Conference on Spacecraft Structures, Materials and Environmental Testing, Noordwijk, The Netherlands, 20-23 March, 2012.

[13] R.R. Craig, Structural dynamics, an introduction to computer methods. John Wiley \& Sons, 1981.

[14] R. Sedaghati, Y. Soucy and N. Etienne, Efficient estimation of effective mass for complex structures under base excitations. Canadian Aeronautics and Space Journal, 49(3):135143, 2003. 